\title{
Internet Of Things: Smart Ubiquitous Architecture of Intelligent Transport System
}

\author{
Muntaha Saleem \\ Queen Mary University of London
}

\author{
Dr. Stefan Poslad \\ Queen Mary University of London
}

\author{
Dr.Waqar Asif \\ City University of London
}

\begin{abstract}
By 2020, there will be more than 24 billion smart devices connected in Internet of Things (IOT). Tremendously augmented motorization, population and urbanization has not only brought us many amenities but also has increased traffic congestion to its limits. In this paper we used IOT to design an efficient and congestion free Intelligent Transport System (ITS).A lot of research is done to either improve or change any one aspect of ITS at one time. This paper demonstrates every aspect or features of an efficient ITS. The purpose of this research is to provide developing countries a detailed and easy to follow ITS architecture, so that they can create an ITS for their populace.
\end{abstract}

\section{INTRODUCTION}

The Internet of Things (IOT) is a network of smart devices embedded with electronics, software, sensors and network connectivity which enables these devices to collect and exchange data [1]. The IOT is an innovation of exchanging data with smart devices without any human interaction. Worldwide system for mobile communication (GSMA) anticipated that by 2020 more than 24 billion devices will be connected to each other which will yield a benefit of 1.2 trillion USD for communication companies [2]. It will impact many application domains, from agriculture to industries, finance, energy and transportation [3]. Immense inflation in urbanization and population did not only bring us many conveniences but also increased traffic congestion to the brink of despair.

This Traffic Congestion has also caused severe reduction in the efficiency of the Transport Infrastructure around the globe. Moreover, the traffic congestion has disastrous impact on economy, environment and society [4]. It also has tremendous impact on the increased travel time, fuel consumption, high cost and air pollution. With the increase in the traffic congestion throughout the world, advanced traffic control system for buses and trains or for a combination of these is extremely needed. A lot of research has been done to analyze or improve any one or few aspects of a traffic system (at one time).

The Bucharest Traffic Management System (BTMS), in Bucharest, Romania implemented a ITS system in 2014. They compared the data collected from the implemented ITS in 2014 and its test project in 2009 which showed significant fuel savings and pollution reduction. But even in this study, not every aspect of ITS was considered like re-routing the traffic dynamically in case of congestion [5]. In another ITS research, an analysis on only the reliability of communication channels was carried out by considering redundant architecture and periodic tests for resilience. The resilience of communication channels could be increased by using duplicate telecommunication equipment. But even in this study, the architecture of ITS was not discussed [6].

In Russia, another research only focused on improving the urban transport system efficiency and logistic processes by introducing water passenger transport i.e. water taxi or Primorskaya Line [7]. In Russia, building a Multimodal Intelligent Transportation System (MITS) 3.0 was proposed. But there were few creation and development problems e.g. intellectual transport infrastructures, cognitive transport security systems and smart transport logistics [8].

The only common problem with these researches are that there was no research or explanation on complete architecture and design of an Intelligent Transport System (ITS). There is a need of such an ITS which can not only reduce congestion but can also increase efficiency. Such an ITS should also be able to have good impact of any country's economy. The goal of this paper is to aid developing countries in creating a successful and efficient intelligent transport system in their country. This paper demonstrates a complete and in detail ITS architecture which could prove useful for developing countries. Another purpose of this paper is to use Ubiquitous Computing and demonstrate a complete architecture of an ITS which can not only increase efficiency and resilience but also decrease congestion.

The first section explains the basics of Ubiquitous Architecture and Smart DEI model of ITS. The second section demonstrates working model of various features of ITS. These features are: use of automated positioning technology, to find route of vehicles, to find travel time for designated pick up points, to show transport arrival routes/current location on mobile phones, to anticipate schedule change and re-route and re-schedule dynamically, to provide information about the capacity of the transport system, to find solution to the problem of transport service render versus schedule delay, to integrate road tube and train maps, to automatically detect buses, to do automatic tagging of locations and to increase the system capacity.

\section{UBIQUITOUS ARCHITECTURE OF ITS}

The ubiquitous computing is used to describe Information and Technology Systems (ICT) that enable information and task to be made available everywhere and to support intuitive human usage, appearing invisible to the user. There are five properties of ubiquitous system: implicit human computer interaction, autonomous, context awareness, intelligent and distributed. The architectural design of ubiquitous system is 
known as smart DEI model. It consists of smart devices, smart environment and smart interactions. Smart devices are multipurpose devices which operate as a single portal and can access multiple applications which are located on servers or other devices. Smart environment is a set of networked devices which are connected to the physical world. Smart interaction are coordinated, semantics and convention based interactions driven by dynamic organizations [9]. In this pa- per, we used ubiquitous computing in designing the various layer of ITS architecture.

\section{A. Smart Design Model}

There are three architectural design patterns of the ubiquitous system which are: smart devices, smart environment and smart interaction. This system is also divided in these three sub systems. Following is the flow chart and brief explanation of the smart DEI model:

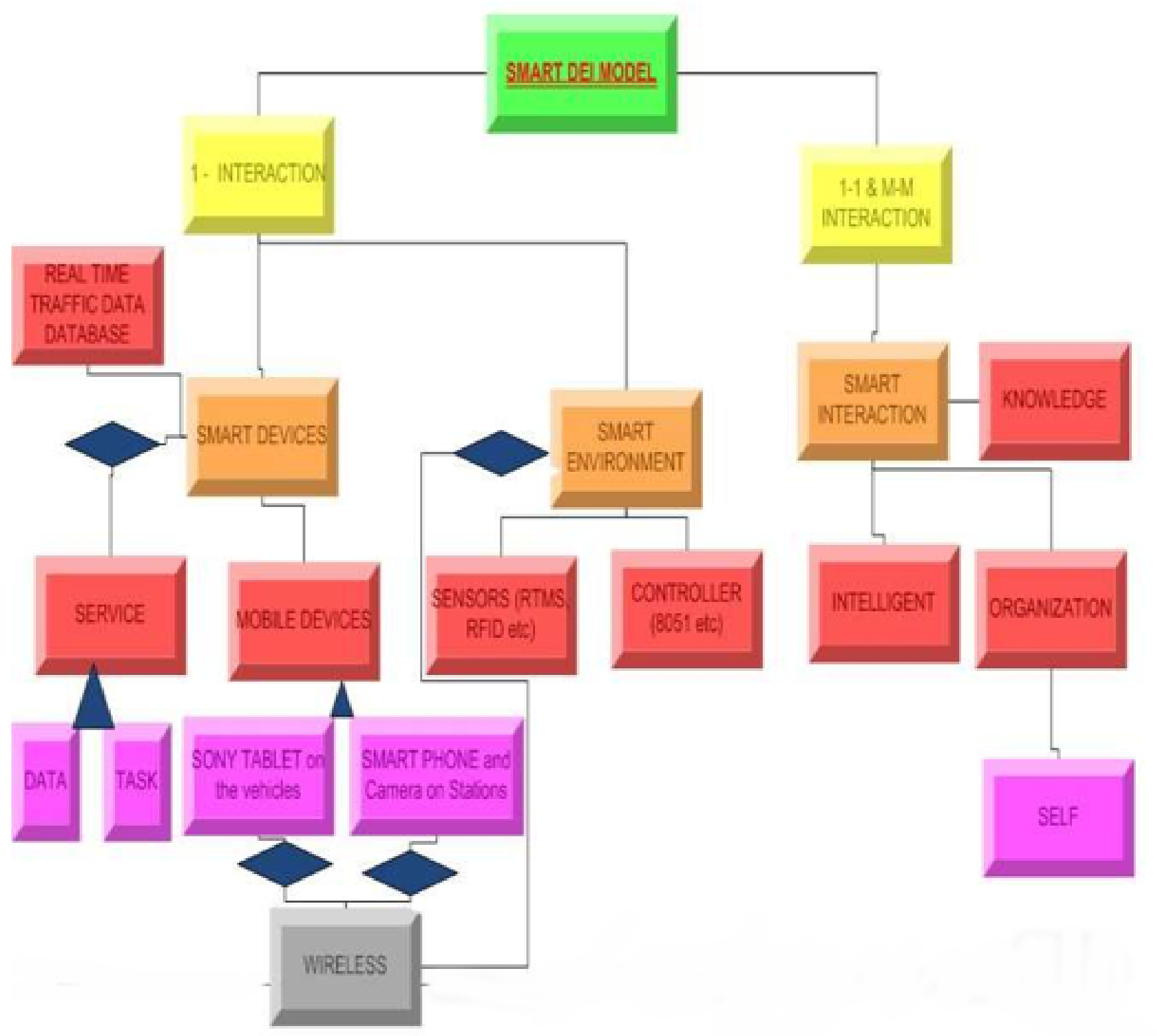

Fig-1 Smart DEI Model of ITS

This system has both kinds of interaction one to one (while transmitting messages to the vehicles) and many to many as well (while answering the user query). In one to one reaction, there is smart devices and the smart environment. Moreover, in many to many interaction, there is a smart interaction.
In an ordinary traffic system, there are four basic nodes of the infrastructure which are: vehicle, pickup points, traffic control center and communication network. This system also has these components, the only difference is the type, specification and the functions of these components. Fig- 


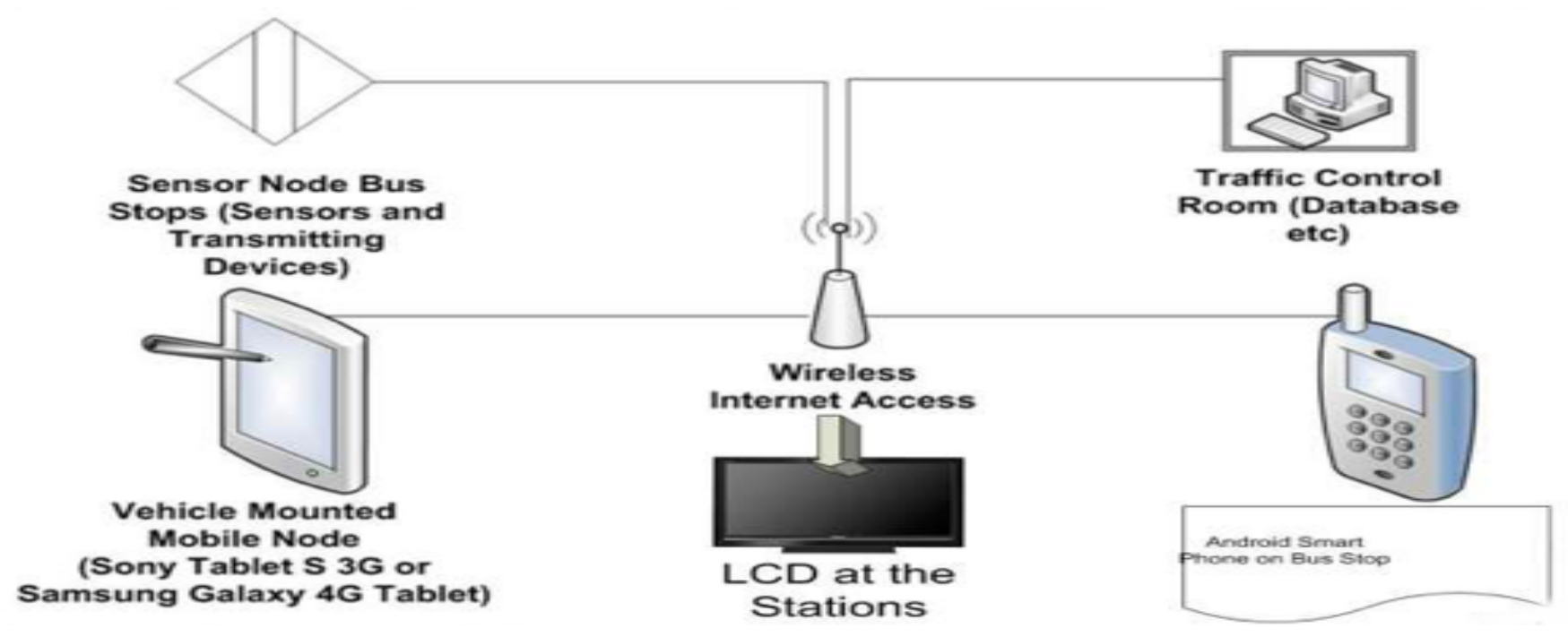

Fig-2 Basic Hardware Structure of ITS

In this ITS there are only five nodes to reduce the work and maintenance load.

1) Node 1: First node is tablet with either $3 \mathrm{G}$ or $4 \mathrm{G}$ technology which could be mounted on the vehicle (bus or train). So that the wireless communication from one node to another would be extremely fast and reliable. There are various different models of tablets (with $3 \mathrm{G}$ or $4 \mathrm{G}$ support) in the market which could be used for this purpose. For this ITS, Sony tablet S $3 G$ and Samsung Galaxy 4 G tablet were considered. In practical any one could be mounted in the vehicle (bus / train).

2) Node 2: In this node the smart phone could be mounted on the stations to interact with the user to inform them about real time transport information. As for the customers, they could have any smart phone or 3G phone. This node could be portable. Some of the requirements of this system could be fulfilled by either $2.5 \mathrm{G}$ or $3 \mathrm{G}$ mobile handset. But to meet other requirements the user must have the smart phones.

3) Node 3: The Traffic Control Room could be one to many (1 $\mathrm{M})$ or many to many (M M) smart interaction. In this node there would be a monitoring system and a database of Real Time travel data. In this node the measured traffic data would be updating after every regular interval. Then this data could be either passed on to the node $1 /$ node 2 or it could be used to calculate new routes / schedule and travel times etc. Then there was a passive monitoring system in this control center which could alarm if something goes wrong either in the infrastructure or in the physical environment e.g. road or train tracks.

4) Node 4: The node 4 was sensors which could be responsible for the measuring and communicating the real time travel data within the ITS structure. There could be sensors like RTMS to detect the speed, arrival time and Location of the incoming vehicle on the bus stop etc. Then again to make life easy for user and the transport control system, there could be RFID card reader and the Android Operating mobile phone could be fixed over the bus / train stop. The basic need of these two was to provide the real time traffic data to the user and drivers as well.

5) Node 5: The connectivity of all the other nodes with each other could be provided by the built in feature of the $3 \mathrm{G}$ in the tablet. This is extremely fast and reliable wireless mode of communication for transmitting the travel data.

6) Node 6: The sixth node could be the LCD which could be mounted on every station to display real time transport information and answer the user query about the system capacity.

\section{B. Smart Environment}

In this ITS, there could be only two smart environment components which were sensors and controller. The sensors come under the static devices which could be embedded on the bus or train stop. These sensors could sense monitor the speed, location and arrival time of the bus / train. Similarly, the controller could be a static device, embedded on the bus / train stop forwarding this data to the control room which could be fixed on the bus. This controller could keep an eye on the real-time traffic data and when something goes beyond the accepted limits, it would automatically report to the control system. In other words, in this system, controllers would adapt control according to the input to them. This work flow is already shown in Fig.2. In this system, the bus driver would tag or annotate the locations which could cause delay in schedule or road blocks etc.

\section{Smart Interaction}

Depending on the certain input and output or requirements, the system could perform different kinds of smart interaction. This ITS system integration mostly depends on the development, integration and interaction of Android Operating 
System application development, Websites development and installation of devices on the bus / train stops.

\section{INTELLIGENT TRANSPORT SYSTEM ARCHITECTURE}

In this paper, the Ubiquitous Properties and DEI Model was designed for Intelligent Transport System for bus and train. For bus, this ITS could do location determination, route determination, travel time consumption, real time vehicle arrival time, tagging locations which can cause delay in schedules, re-routing and re-scheduling of the system, answer customer query about transport capacity, cater to delays with conflicting goals such as to pick extra customers, integration of routes for different types of modes. Following is the detailed design of every feature of this ITS.

\section{A. Automated Positioning Technology}

The objective was to increase capability for the inter operable Mobile Devices, so instead of using a GPS device separately we chose such a tablet which has built in GPS. So that when it is fitted in the bus / train then it could automatically display the current Location coordinates and would change in real time. This was a context aware physical ubiquitous computing property because of detection of location of the bus / train in the system. This tablet was also selected because of its small size and shape because it was possible to accommodate it anywhere in the vehicle. Moreover, as in London traffic system, there is a huge set of box that plays a role of reading an oyster card and then deducting the travel fare from it. But instead of that whole box, we could use a simple tablet attached with a small card reader to perform the same function. This was one of the another reason of choosing the tablet as a vehicle mounted device.

\section{B. Route of Vehicles}

The driver before starting the journey would enter the start and end positions in the tablet application along with his/her code. Then the Android application on the tablet would be able to use Google maps and its built in GPS to find the route and (pre-selected route for the specific bus number or train number) display on the screen. For the bus driver, this interaction was 1-1 and explicit human computer interaction. This service was provided on demand from the user or drivers. This was active context awareness property of the system because it automatically detected the user current location and informed the driver about the next move or turn etc. For the user, he / she could get route or travel time information from the ITS in four different ways e.g. SMS, Call, Website and the Mobile application. In travel time scenario, the method of user query was same as in the route query, but the only difference was that there would be some other procedures that needed to take place before sending the information (answer to his / her query) to the user. So for both requirements, the four different ways are explained as:

1) SMS: The user should have either $2.5 \mathrm{G}$ or latest technology mobile phone. The user would send the current location name / coordinates, destination name / coordinates and the option number (which specifies the type of information he needed) to the designated number which was given by the ITS. These coordinates would go through the GSM / CDMA network and reach the ITS Database. After acquiring the data from there, the ITS system would automatically generate the SMS with the route or travel time information (depends on the query) and would send it back to the user by GSM / CDMA network (Fig-3).
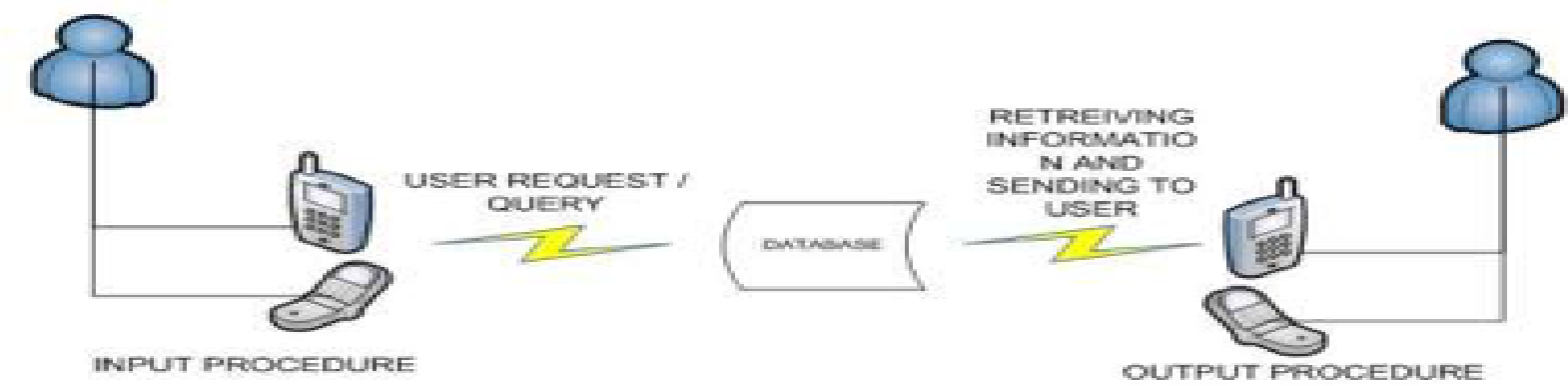

Fig-3 Flowchart of Information via SMS
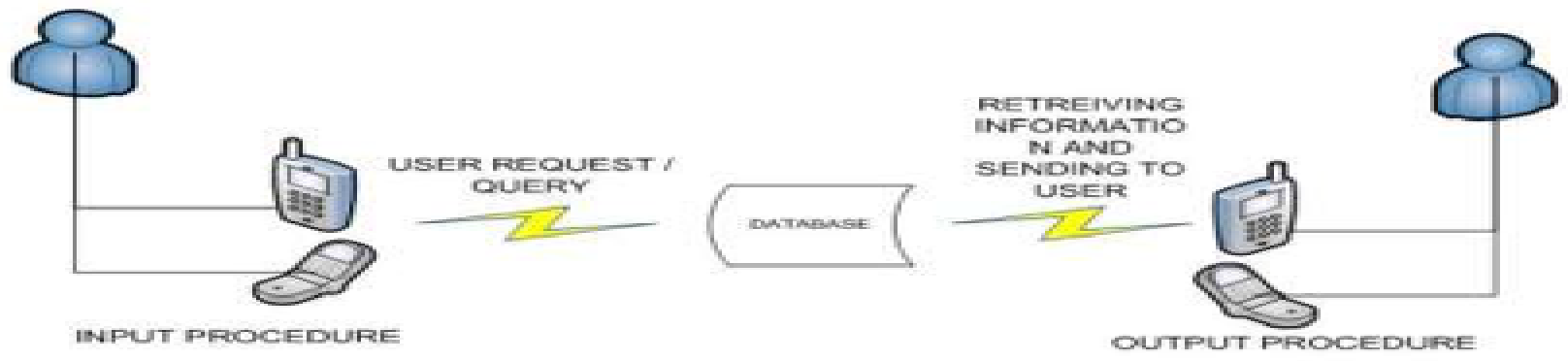

Fig-4 Flowchart of Information via Call 
2) Call: Similarly, if the user wanted to make a call and find out the route or travel Time Information for either bus or train. There was an automatic voice call receiver algorithm used in the system to answer the query of user. After the call was established, upon asking the user could enter the option for the service he wanted e.g. 1 for route and 2 for travel / time. If the user selected the option 1 then upon asking he could enter the post code / coordinates or name of the Start and End Point. As a result, the software at the server end would get the required data and answer the user query. But if the user selected the option 2 then, upon asking the user entered the bus number or train number to get the answer (Fig-4).
3) Website: The user would need to get the route from the website especially for those who wanted to access via computers. For them there could be a website included in the design. Through which the user could enter the start and end positions to get the routes online. This command would first go to the server and then go to database and access the ITS database information and then come back through exact same path and reached the user computer. Similarly, if the user wanted the travel time information, then he could enter the bus or train number in the online query form to get the instant result. Fig-4 is the diagram for the procedures that could take place if someone wanted to access the information from the website(Fig-5,6).

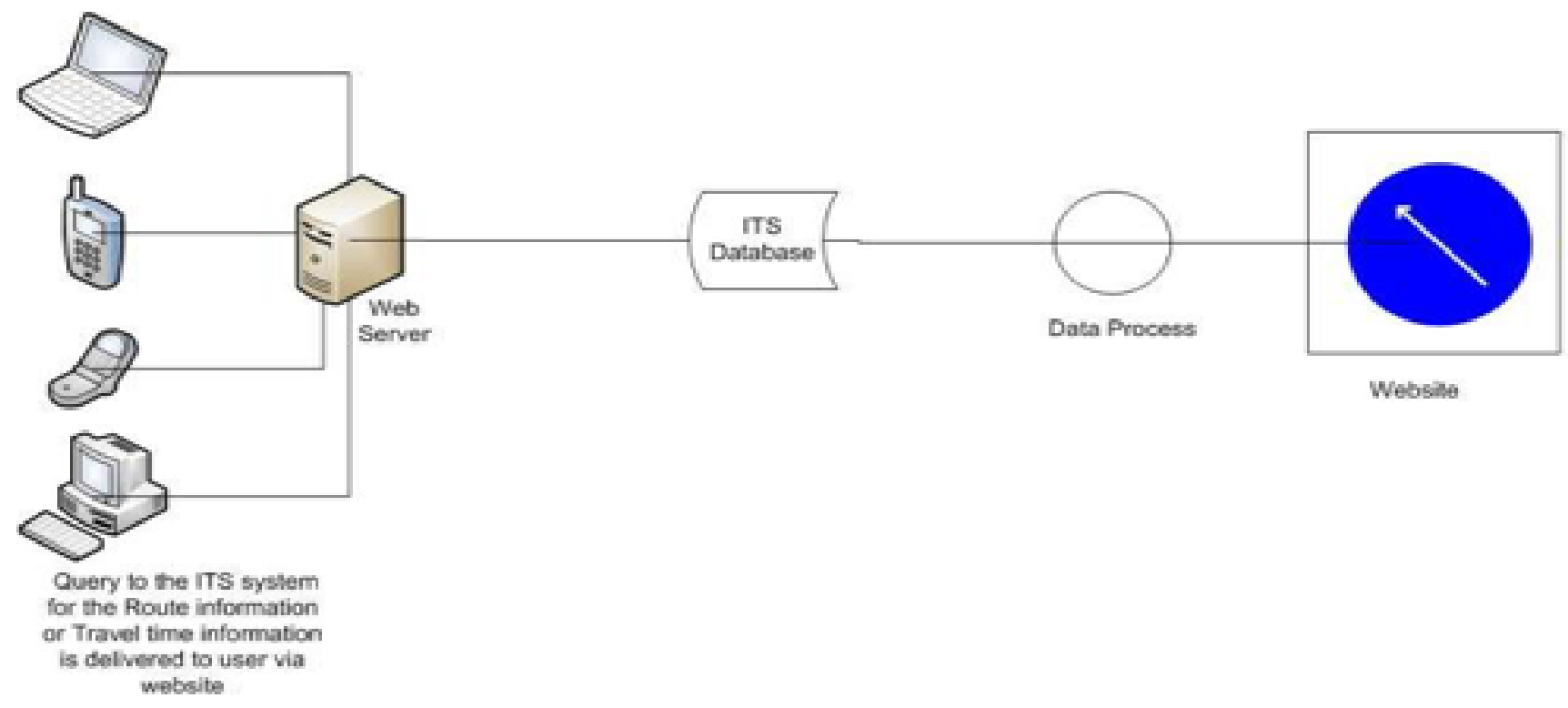

Fig-5 Input Flowchart of Information via Website

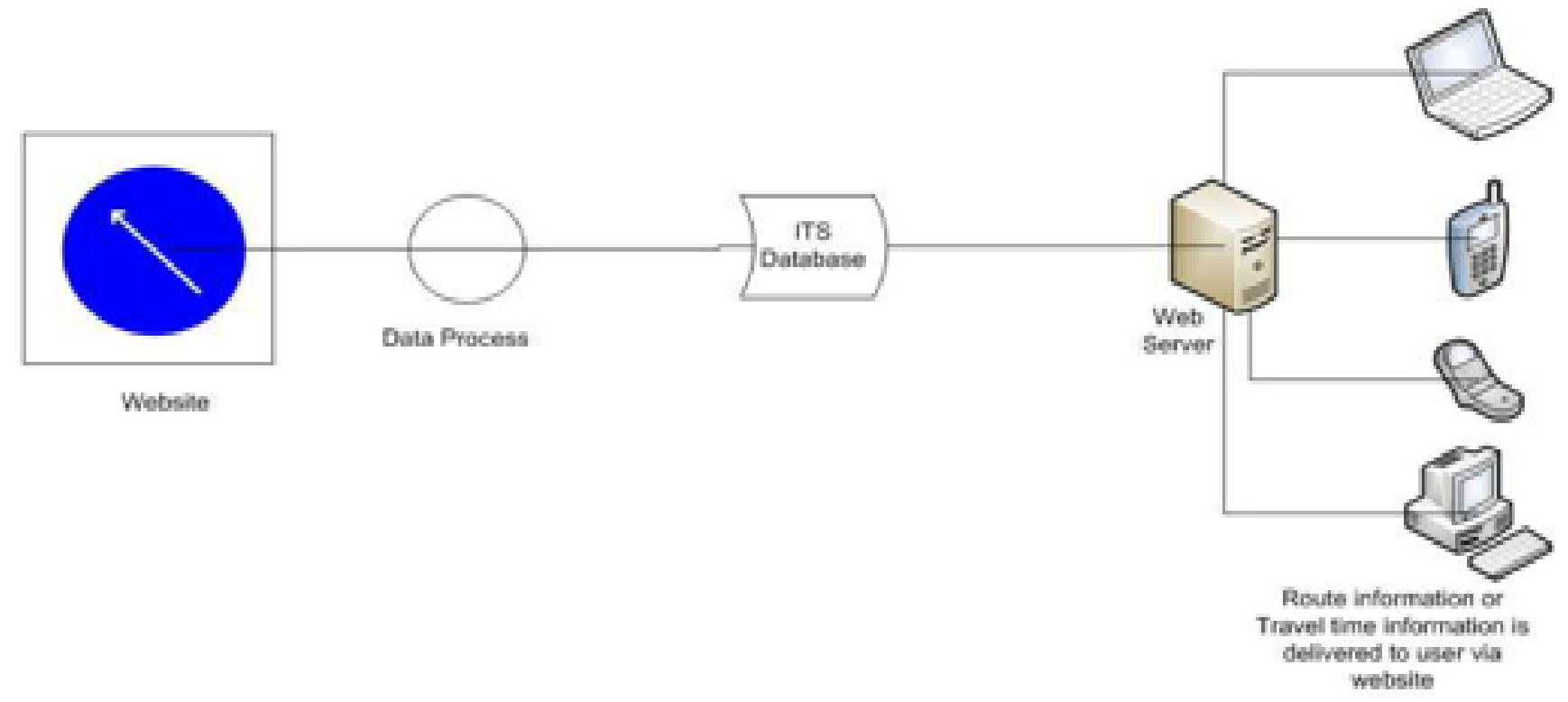

Fig-6 Output Flowchart of Information via Website 
4) Mobile Application: This could be an easiest way to achieve the traffic Information like the Routes, travel times etc. The smart phones would send the query online to the ITS database by filling a form available in the application and would get the required results from the database in the same way as in the website scenario(Fig-7,8).

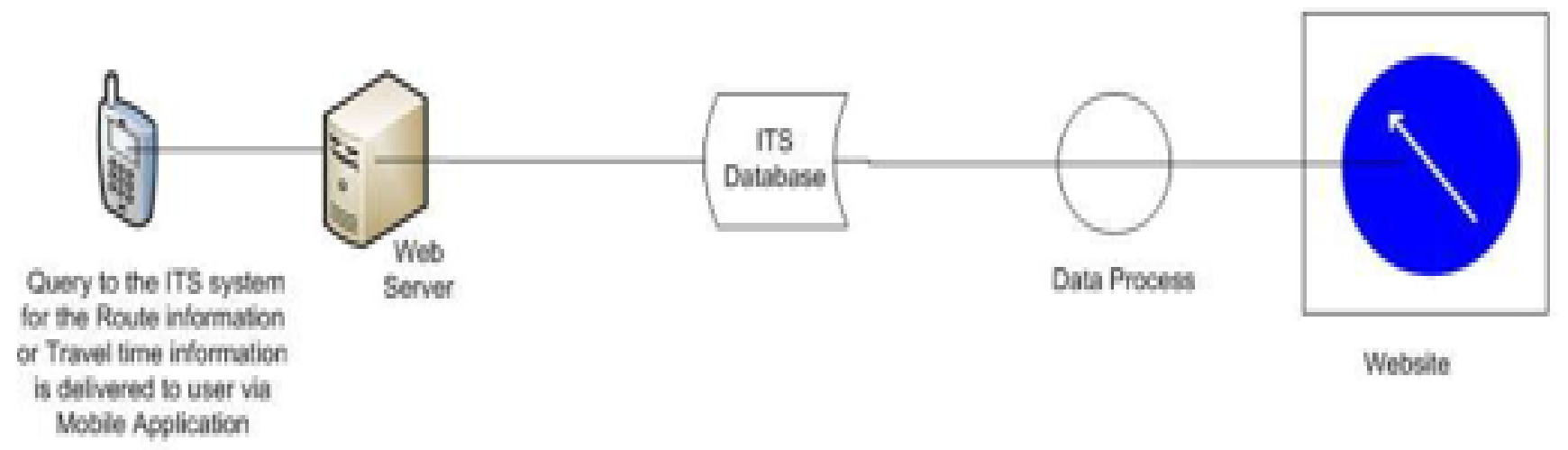

Fig-7 Input Flowchart of Information via Mobile Application

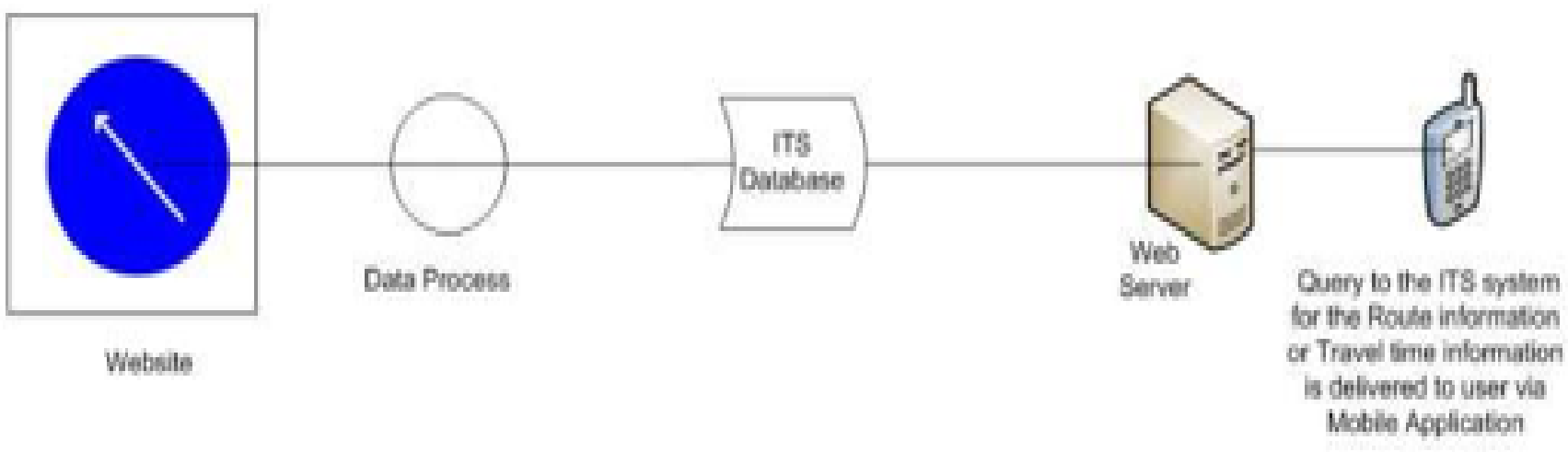

Fig-8 Output Flowchart of Information via Mobile Application

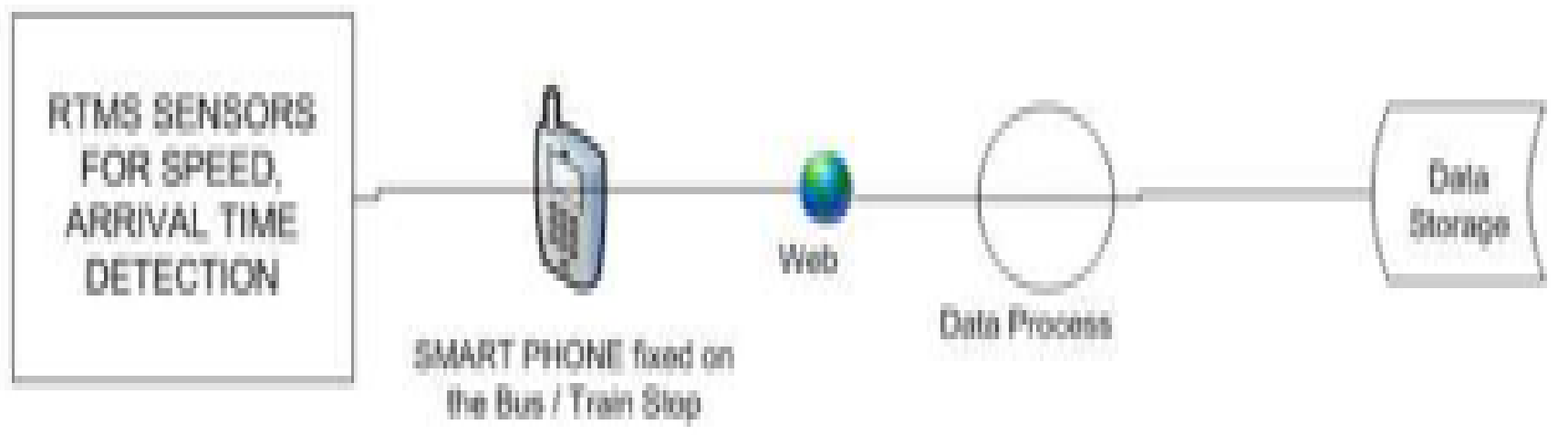

Fig-9 Flowchart while accessing Information

\section{C. travel time for Designated Pick up Points}

There would be RTMS sensors fixed on the bus / train Stop which would measure the speed, arrival time, and the time taken to travel to the final destination based on the average values which could be stored and updated in the data base after regular interval of time. As the arrival time 
would be either displayed on the sign board or sent to the user terminal (smart phones, websites etc.), therefore it is an on demand asymmetric remote services because of less upload and more download from the database. Moreover, it is a synchronous basic smart environment interaction. The procedure to calculate the travel time information is different. If it was to provide to the user than the same four ways are applicable here i.e. SMS, call, web and mobile application.

\section{Transport Arrival Routes and Current Location Informa- tion on Mobile Phones}

The bus / train stop would have the LCD and Smart Phone mounted on it along with the RFID tag reader [10]. The LCD would display the arrival time, specific route and location of a vehicle on it for each bus for an interval of $30 \mathrm{sec}$ (Fig-10). The LCD would receive this information over the internet connection from the database. In other words, the RTMs would calculate the travel time and send to the database to store and then to be forwarded to the LCD to display. The purpose of RFID tag reader was to keep the smart phone lock as long as any user used his RFID travel card to unlock the smart phone. For some reason if the user wanted to see the information about the certain bus / train number than he / she could use their Travel cards on the RFID tag reader. Once verified by the Tag reader that the user has a valid transport card (money to travel did not matter means the user could still use service if they have no money on the card), it would unlock the smart phone. Then the user could enter the bus / train number on the available screen and press Enter. After this, smart phone would send a command to the Database over the $3 \mathrm{G}$ connection to display the required bus / train information on the LCD. When the required information was displayed for 30 seconds, the Smart Phone would automatically lock and the tag reader would reset.

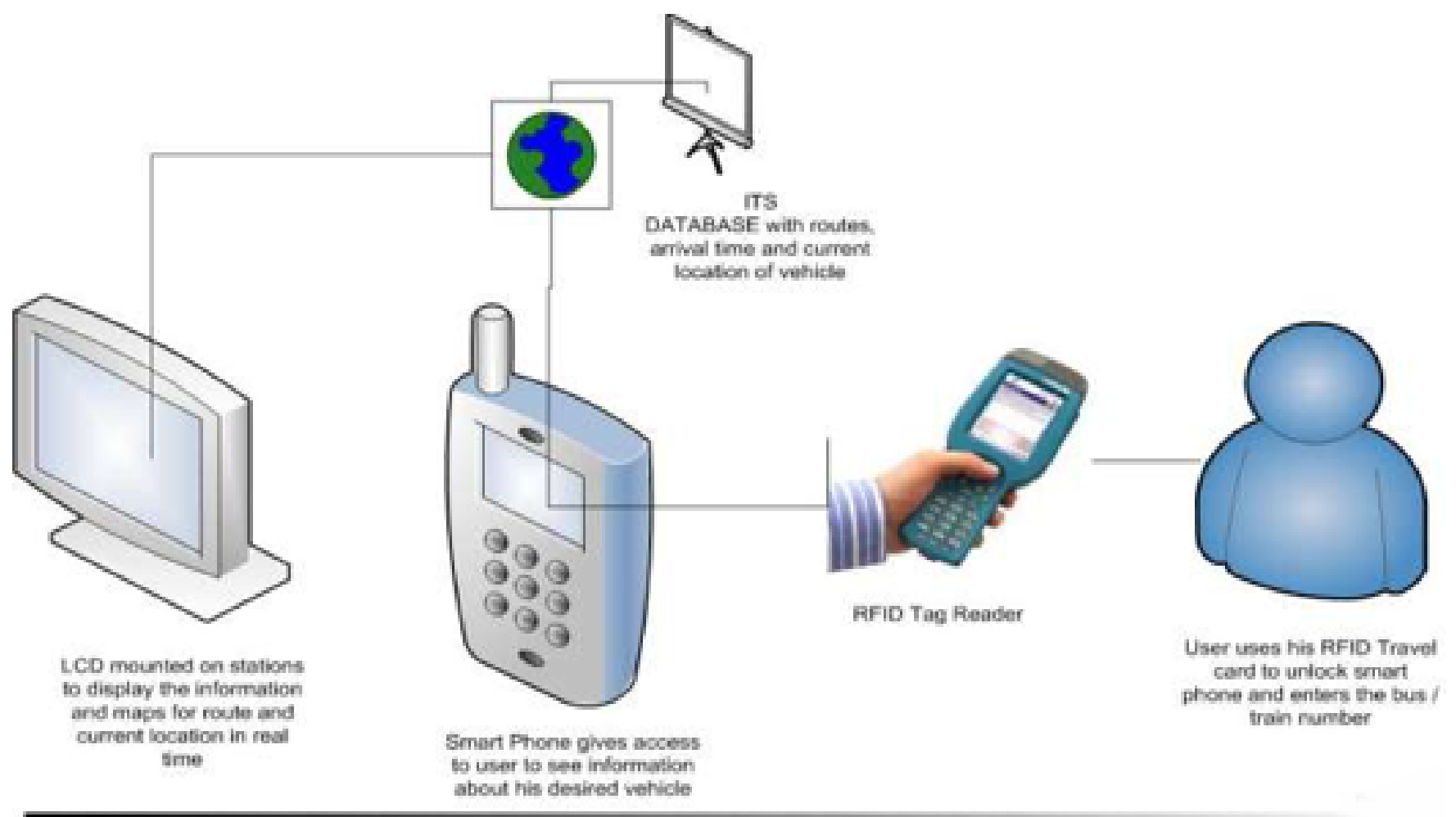

Fig-10 Transport arrival, routes and current location information on mobile phones

\section{E. Anticipating Schedule Change and Re-Routing and Re- Scheduling Dynamically}

To solve the problem of drivers reporting a faulty or blocked route etc., the Android Application would have a specific function for this purpose. The driver would only need to press a touch button on the Sony Tablet and could enter the coordinates of the current location and then press send. Then tablet would automatically update the information in the database and tag that location as not available. So that while planning any kind of route either for bus driver or for the user the system could not consider this stop and find the route without that stop. Then the system would automatically Reroute or Reschedule for the driver and display the information to all the other tablets on the buses with in the 10 miles on the same route until that old route could clear of trouble. But for the train it is not possible to change the tracks, therefore upon facing the problem on a track all other trains would halt or terminate at the previous stations depending on the time it takes the problem to be solved. Then if the problem was severe then the lines 
could terminate for indefinite time until the problem could be solved. For the rerouting and the re-scheduling of in buses, the RDIN-R method could be used because of its efficient results shown in Munich University [11].Similarly for train rerouting and rescheduling, Tabu Algorithm could be used because of its ability to handle the real time traffic data [12]. Fig-11 12 are detailed structure diagrams which can explain this procedure in more detail.

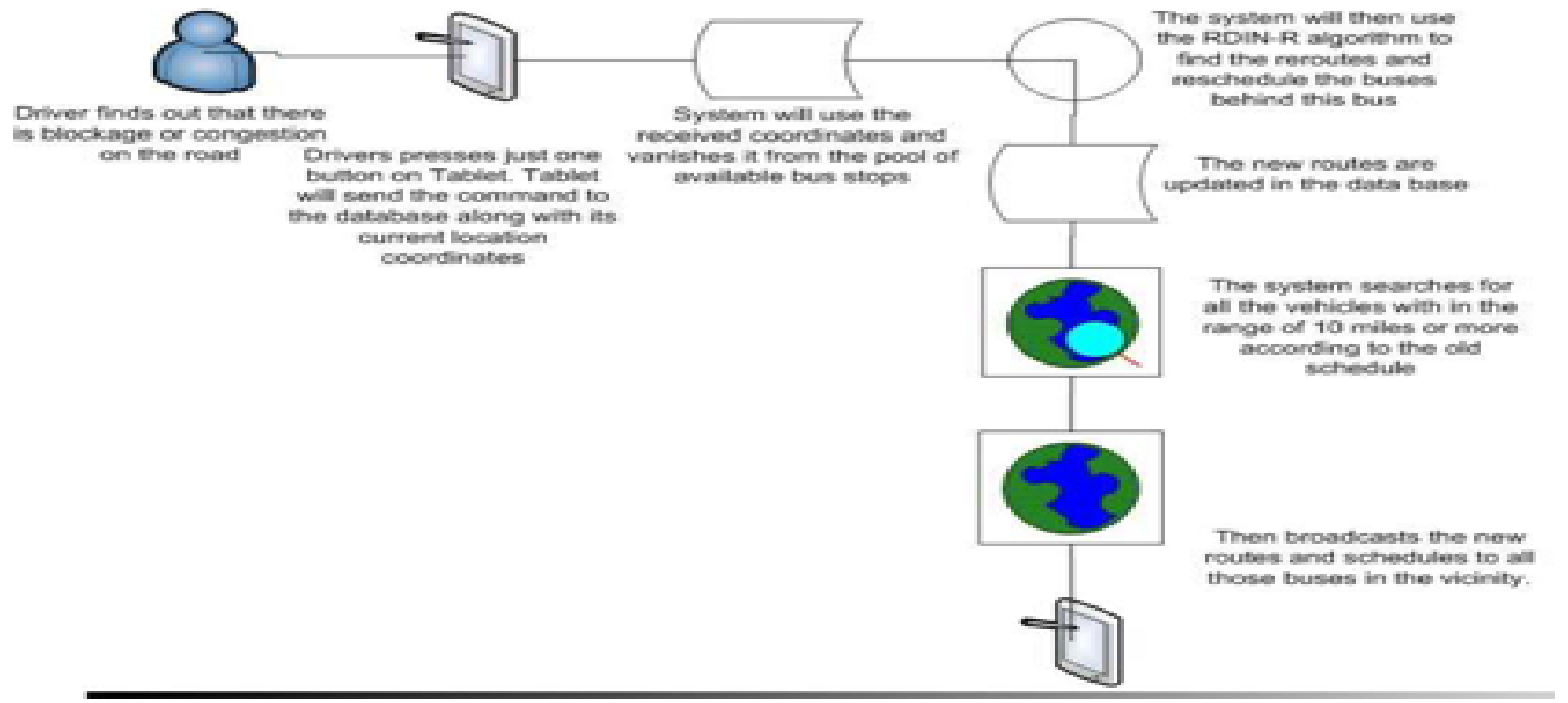

Fig-11 Anticipate schedule change for bus

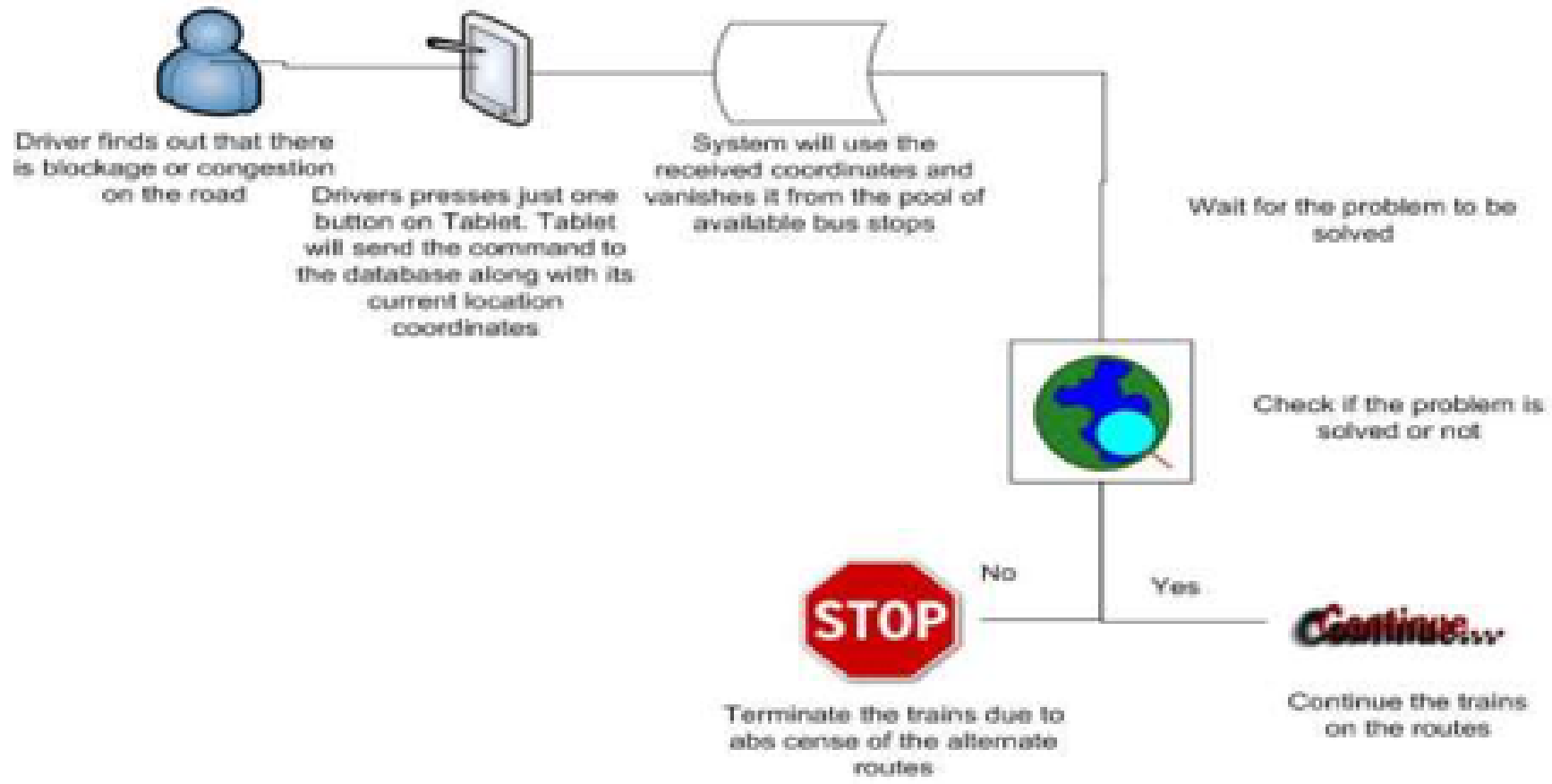

Fig-12 Anticipate schedule change for train

\section{F. Information about the Capacity of the Transport System}

There was a Smart phone, LCD and RFID tag mounted on the stations which could be used to get the information about the capacity of transport service (Fig.13-14). The only additional thing here was the algorithm due to which the system found out that whether there was an enough capacity to fulfill the user demand or not. When the user used his RFID Traveler card to unlock the Smart Phone and gave the number of customers waiting on the stop A or number of goods at the pickup point $\mathrm{A}$ to the system on the screen for Transport Capacity. Then the Smart Phone would transmit this number to the system over $3 \mathrm{G}$. Then the system would first divide the total number of customers by the total bus / train capacity (number of seats) and find out that 
approximately how many buses would be required to meet the demand. The algorithm would automatically truncate the floating point and rounds off the answer to the largest number to avoid any trouble. Then the system would determine the customer demand on the stops before the stop A on the same route by the Image Processing. We supposed the name of Algorithm to be "Image Algorithm". After analyzing the previous bus stops (if there are no customers on some bus stops then system could skip it), the system decided whether it could skip any of them or not. If the answer is yes, then it would ask the buses behind bus A to skip those selected bus stops to meet the demand on stop A. Also the system would display the message "Yes we can meet the user demand more buses are on their way". But if by any means there was no bus stop the system could skip, then it will just display the message "Sorry but due to huge user demand there is a problem, the buses will arrive soon".
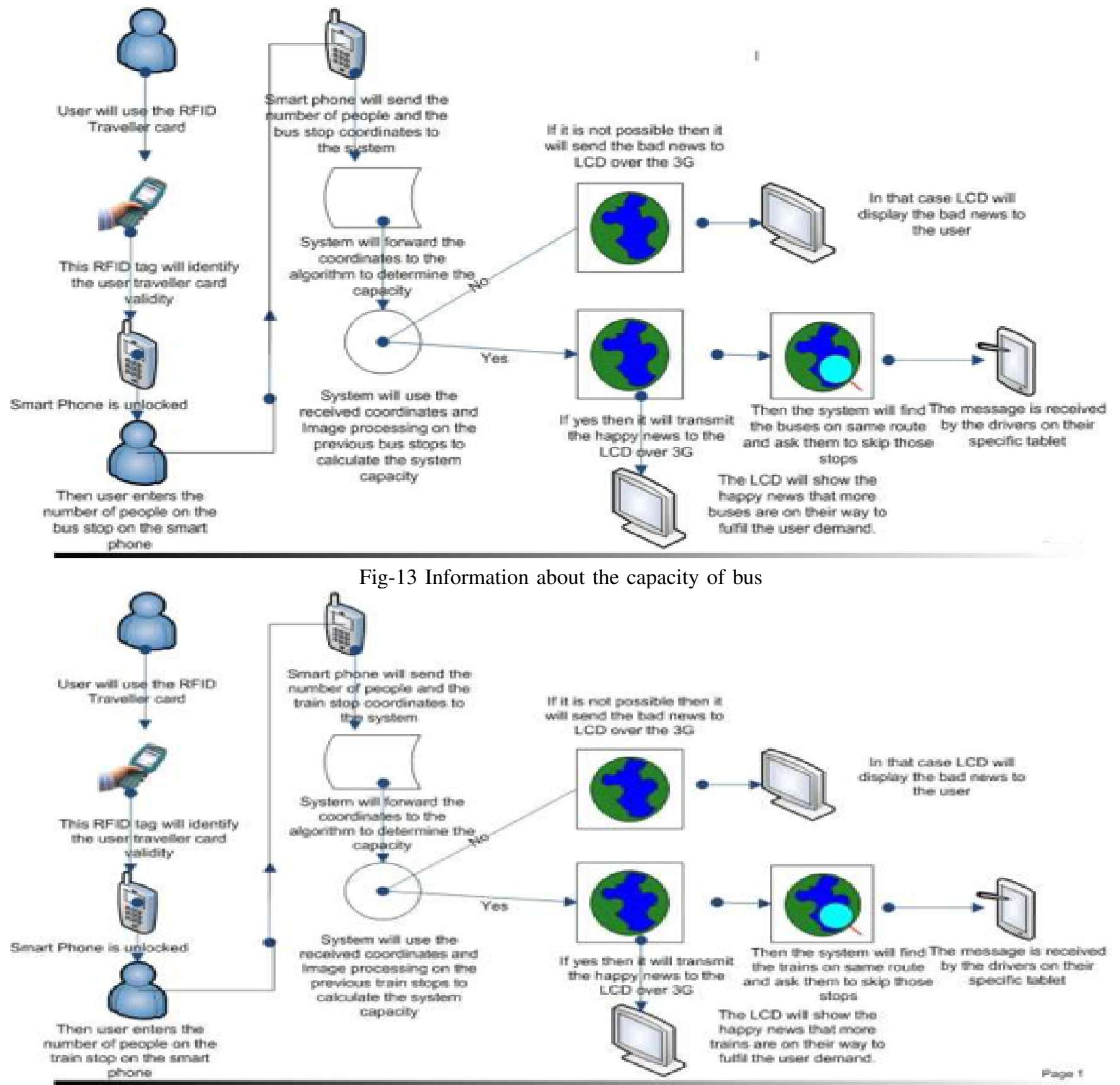

Fig-14 Information about capacity of train

\section{G. Solution to the Problem of of Transport Service Render versus Schedule Delay}

In this ITS both quality and revenue generation was balanced. For this neither the bus should be delayed nor the quality should be compromised. Therefore, the easiest and cheap way to sense the presence of person on one seat was to use the sensitive light emitters beneath the seat along with 
the LED as a light detecting device at the exact top of the bus roof in the inside so that receiver could detect the infrared at the 90 degrees. Attach a micro controller e.g. Atmel 8051, which could keep an eye on all of the sensors and find out if any seat was occupied (if seat was occupied the LED as a light detecting device would not receive light). If micro controller concluded that all seats were occupied than the doors should automatically close by the use of motors and the announcement would be made on the speaker. This method could be same for both cases of the bus or train (Fig-15).

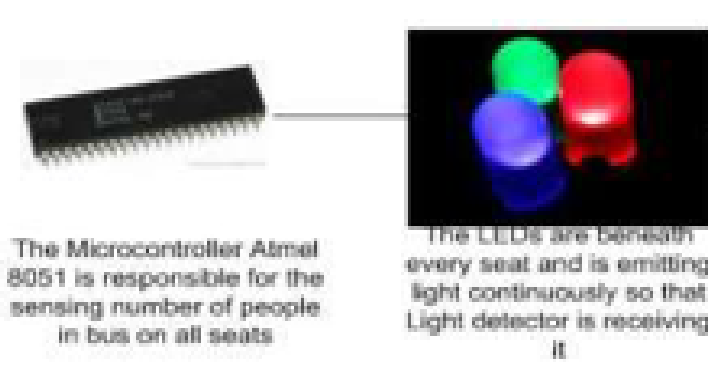

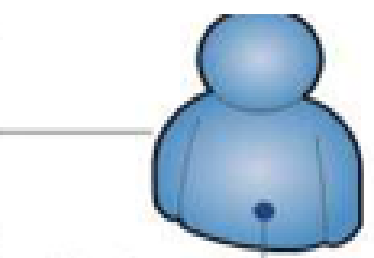

When the user cothes and sit down

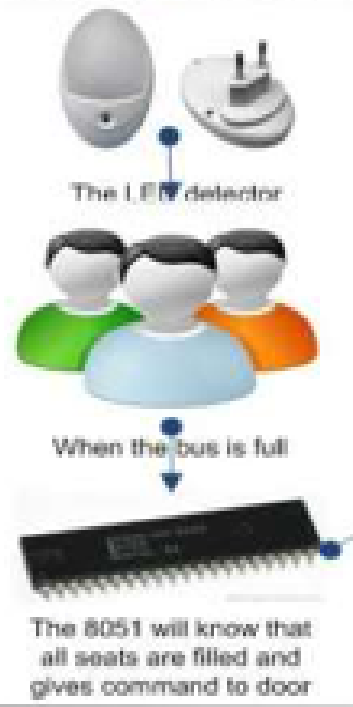

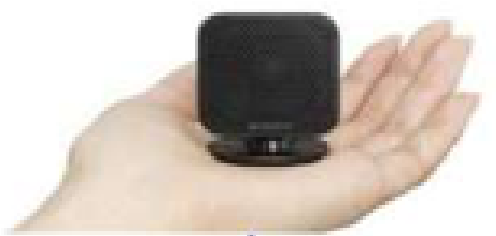

The microcontrolier Aliso plays a audio of announcing the message about the bus being flllad on the speaker.

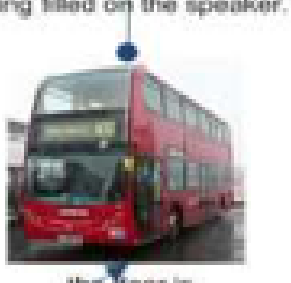

the boor is

cloased automatically whout letting anyone to get in

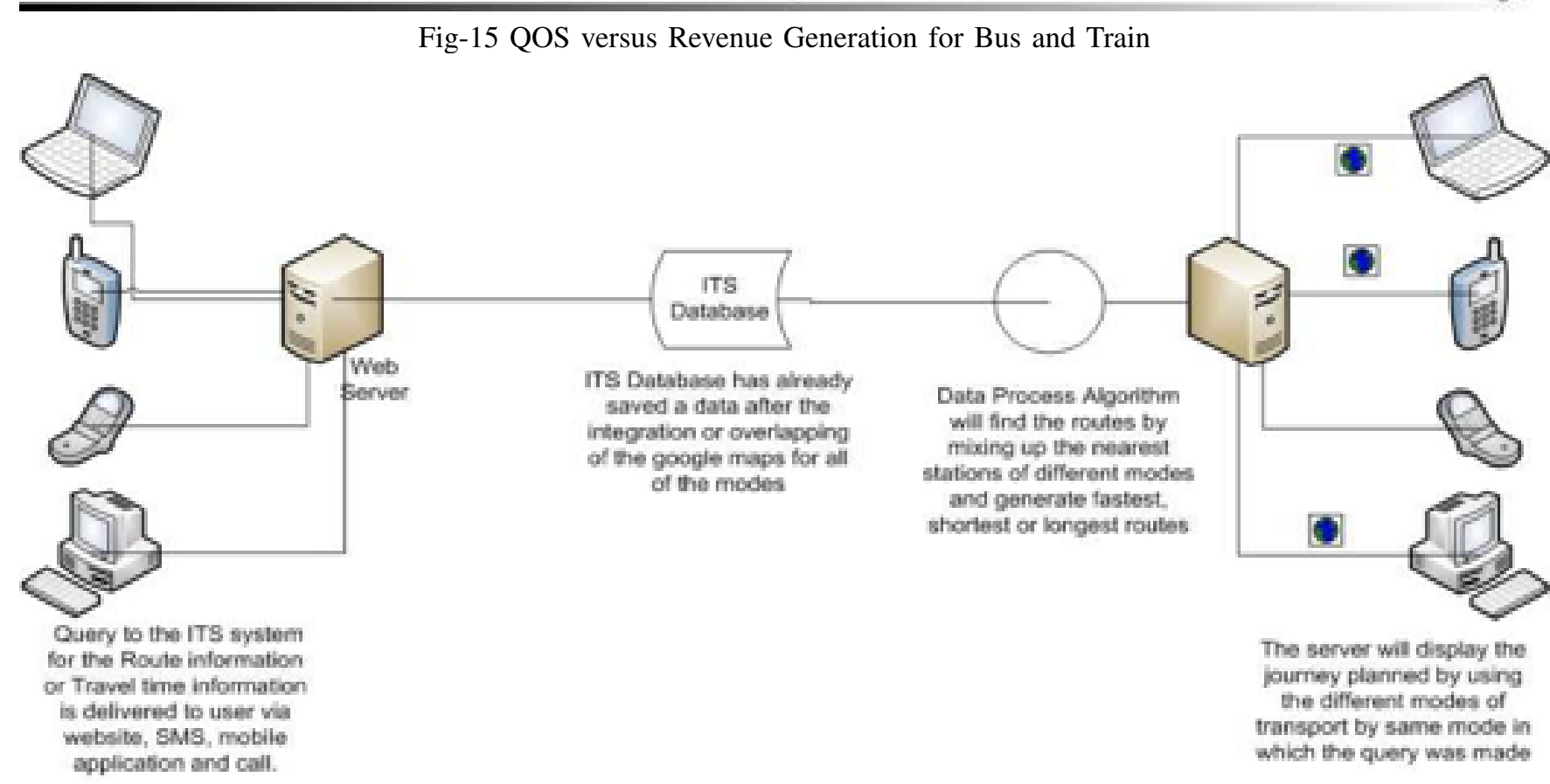

Fig-16 Integration of Routes and Maps

\section{H. Integration of Road Tube and Train Maps}

For this, the main role was played by database and the method proposed by " An Interactive Web-Based public transport inquiry System with Real-Time Optimal Route Computation" [13]. The database has the integration of Google maps for all of the modes of transport. The written 
information and data of transport services with respect to real time is also included. Whenever the driver entered the initial or final stop name or coordinates then the system could plan by a common algorithm to find the shortest, fastest and the longest routes types by taking into consideration the nearest stops of any mode of transport just like a journey planner (Fig-16). This was basically a software based requirement of this system which could be met by designing an interactive website and mobile application for the smart phones.

\section{Automatic Detection for Buses Automatic Tagging of Locations}

The bus driver attention should not be diverted at all. If the driver noticed the number of cars and gave the data to the tablet for location tagging, it would not be suitable. Instead of the driver pressing a button to tag a location on the Tablet, there should be alternative of automatic tagging location whenever congestion was detected. This system already implemented cameras on the bus stops to find the number of people so that the buses could skip bus stops if no one was present. The same technique of Image Processing should be used to combine the two requirements by first doing automatic congestion. For this each bus should have the camera to capture video for image processing mounted at the top of the front mirror at the outside. This camera should keep track of the number of License plates of the car at front of it (Fig-17). There should be a certain number of cars that defines normal traffic flow. If the application designed on the Android OS Sony Tablet determined that the number of License plates at front of it is increased than the limit then it would automatically initiate the procedure of the requirement of "Tagging the Locations" without disturbing the driver. In this way the driver's concentration would not be diverted and there would be less danger of accidents and mishaps.

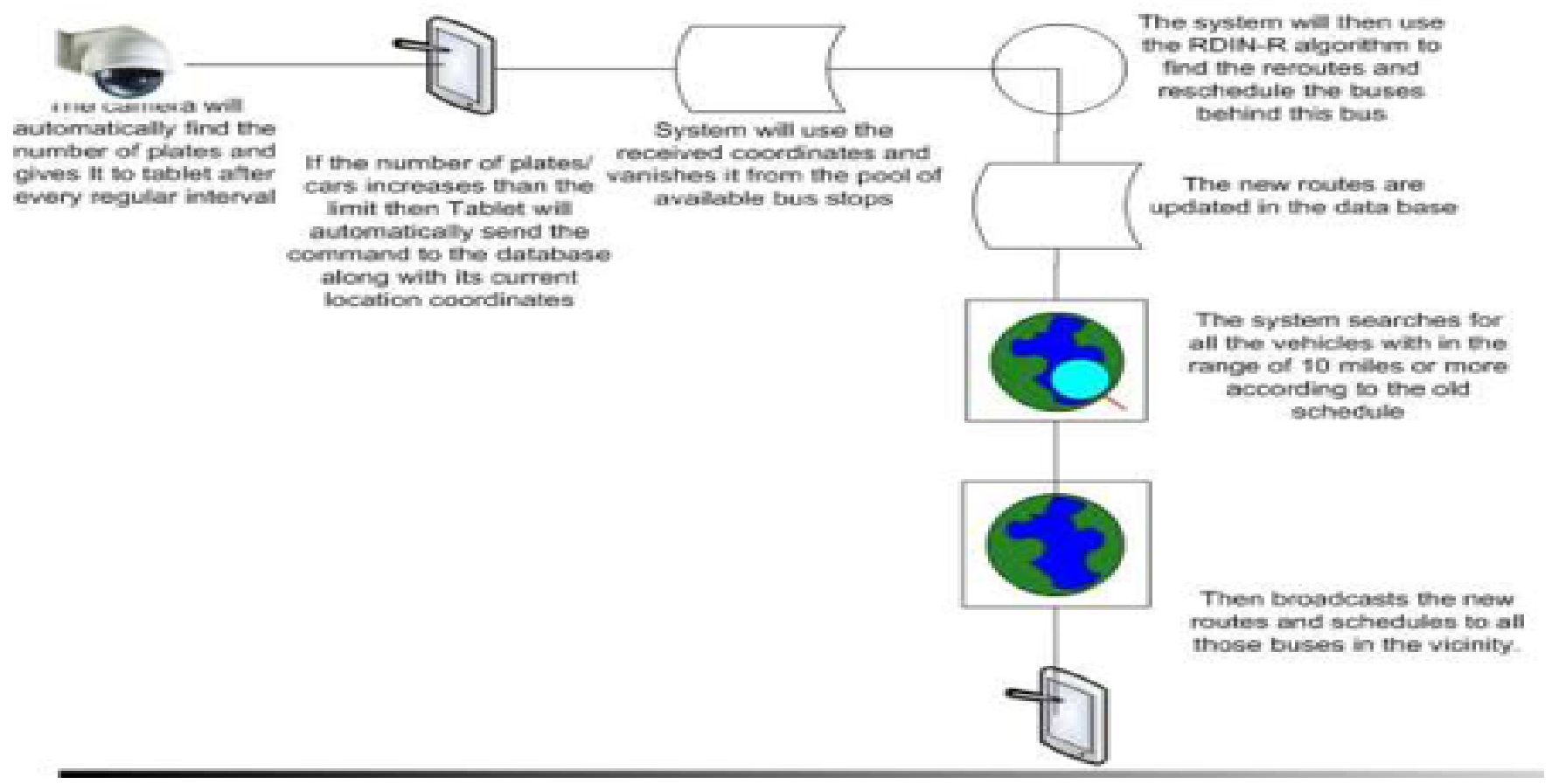

Fig-17 Automatic Congestion Detection

Now the point was which technique should be used to perform such kind of an Image Processing. There was a "A Template-Based Model for License Plate Recognition" proposed in the [14] which could be used for the detection of the number of plates of vehicles and thus could determine the congestion. Sometimes the user needed to know the route, the upcoming bus stops and the live vehicle location while sitting on the bus, e.g. when user did not know how many more stops he has to go to reach his / her destination. For this there could be a LCD mounted on buses to display the information and maps with vehicle location in real time. The ITS Database would provide this information to the LCD fitted in bus.

\section{J. Increase in System Capacity}

For increasing population, the public transport would never be enough, so instead of increasing the services for full time. ITS should support extra services in the urgent scenarios when there was more user demand. In this way, without using extra resources for full time the system efficiency could be increased. As this system displayed a message that it could not meet the user demand because it could not skip any bus stop in one of the above features. In that case, instead of displaying such message the Control Room would order more buses to start on the same route but not from the first stop but from that stop where user's density is huge. But for this, there should be at least 5 or more buses extra in the bus stand for every route. The condition was that this extra 
bus would not pick customers from next stops if there was no crowd on other stops, it should just keep dropping people on the bus stops. As soon as it got empty, it should come back to the bus stand to be available in the pool of extra buses. In that way, not only the buses on the route but also the extra buses would be helpful in providing the excellent Quality and Revenue Generation.

\section{CONCLUSIONS}

This paper concludes by proposing a system which could determine location, route, travel time, real time vehicle arrival time, tag locations which can cause delay in schedules, re-route and re-schedule the system, answer customer query about transport capacity, cater to delays with conflicting goals such as to pick extra customers and integration of routes for different types of modes. The main purpose of this paper was to provide very detailed ITS architecture and design to everyone, so that developing countries may adapt this system and could provide a congestion free and efficient ITS to their people. While there is an advantage from the implementation of ITS, there are however many opportunities to continue scrutiny, research work and application. The next goal in this research is to do simulation of this ITS, standardize the ITS and identify most suitable technology and infrastructure to increase the efficiency of system exponentially,

\section{REFERENCES}

[1] J. Mann, "The internet of things: Opportunities and applications across industries," International Institute for Analytics, Enterprise Research Service, December, 2015.

[2] A. Majeed, "Internet of things (iot): A verification framework," in Computing and Communication Workshop and Conference (CCWC), 2017 IEEE 7th Annual. IEEE, 2017, pp. 1-3.

[3] S. Ziegler, "Considerations on ipv6 scalability for the internet of thingstowards an intergalactic internet," in Global Internet of Things Summit (GIoTS), 2017. IEEE, 2017, pp. 1-4.

[4] G. Muthumanickam and G. Balasubramanian, "A traffic congestion control in urban areas with vehicle-infrastructure communications," in 2017 International Conference on Energy, Communication, Data Analytics and Soft Computing (ICECDS). IEEE, 2017, pp. 29702974.

[5] A. R. Florea and I. M. Costea, "Analisys of improvements the urban transport conditions by using electronic intelligent transports systemscase study: Urban transportation," in Electronics, Computers and Artificial Intelligence (ECAI), 2014 6th International Conference on. IEEE, 2014, pp. 73-78.

[6] I. Kabashkin, "Reliable v2x communications for safety-critical intelligent transport systems," in Advances in Wireless and Optical Communications (RTUWO), 2017. IEEE, 2017, pp. 251-255.

[7] S. Seliverstov, I. Malygin, A. Starichenkov, R. Muksimova, V. Grigoriev, and A. Asaul, "Modeling of megalopolis traffic flows with the introduction of a new line of water intercity passenger transport," in Soft Computing and Measurements (SCM), 2017 XX IEEE International Conference on. IEEE, 2017, pp. 278-280.

[8] I. Malygin, V. Komashinsky, and V. Tsyganov, "International experience and multimodal intelligent transportation system of russia," in Management of Large-Scale System Development (MLSD), 2017 Tenth International Conference. IEEE, 2017, pp. 1-5.

[9] S. Poslad, Ubiquitous computing: smart devices, environments and interactions. John Wiley \& Sons, 2011.

[10] K. Mandal, A. Sen, A. Chakraborty, S. Roy, S. Batabyal, and S. Bandyopadhyay, "Road traffic congestion monitoring and measurement using active rfid and gsm technology," in Intelligent Transportation Systems (ITSC), 2011 14th International IEEE Conference on. IEEE, 2011, pp. 1375-1379.
[11] I. Kaparias and M. G. Bell, "A reliability-based dynamic re-routing algorithm for in-vehicle navigation," in Intelligent Transportation Systems (ITSC), 2010 13th International IEEE Conference on. IEEE, 2010, pp. 974-979.

[12] A. D'Ariano, "Improving real-time train dispatching: models, algorithms and applications," 2008.

[13] L. S. Pun-Cheng, "An interactive web-based public transport enquiry system with real-time optimal route computation," IEEE Transactions on Intelligent Transportation Systems, vol. 13, no. 2, pp. 983-988, 2012.

[14] Z. Salcic, "Agpcs-an automatic gsm-based positioning and communication system," in Second annual conference of GeComputation, University of Otago, New Zealand, 1997, pp. 26-29. 